\title{
DIÁLOGO CON ANDRÉS SÁNCHEZ ROBAYNA ACERCA DEL TALLER DE TRADUCCIÓN LITERARIA DE LA UNIVERSIDAD DE LA LAGUNA
}

Clara CURELL ${ }^{1}$

Universidad de La Laguna

ccurell@ull.edu.es

lara Curell: Se acaban de cumplir veintidós años de la constitución del Taller de
Traducción Literaria (TTL), lo que lo convierte en una experiencia más que consolidada de
trabajo colaborativo en este campo. ¿Cuál ha sido, en líneas generales, la evolución de este novedoso proyecto a lo largo de estos años? ¿Se han visto cumplidas las expectativas que tenías cuando promoviste su creación en la Universidad de La Laguna en 1995?

Andrés Sánchez Robayna: El balance, a mi ver, es muy positivo. Basta repasar la lista de nuestras publicaciones -incluidos los artículos y ensayos aparecidos en el Boletín del Taller - para percatarse del trabajo realizado a lo largo de más de dos decenios. No es cuestión de resumir aquí la historia del Taller, porque es una historia que ha sido contada ya en varias ocasiones, pero sí vale la pena subrayar un par de aspectos que considero centrales. Tu pregunta sobre la evolución del Taller resulta muy oportuna. En primer lugar, yo nunca habría imaginado que lo que empezó siendo un proyecto muy modesto relacionado con la traducción de textos literarios más o menos complejos (de textos, decíamos entonces, «que por su nivel de elaboración o de información estética presenten un grado especial de dificultad o de complejidad») acabaría convirtiéndose en un seminario permanente y que iba a tener la continuidad que ha tenido. Yo pensaba que íbamos a trabajar durante un tiempo y, una vez realizadas algunas traducciones y comprobada en otro contexto la metodología que yo aprendí en los seminarios de Royaumont, la experiencia quedaría en cierto modo agotada en sí misma, pensando sobre todo en la dificultad de la tarea, que exige un esfuerzo sostenido. Las cosas, sin embargo, no fueron así, y pronto me di cuenta de que dejábamos atrás lo aprendido en Royaumont y enriquecíamos la experiencia con otros métodos y formas de trabajo. Pasamos, por ejemplo, de la traducción colectiva a la revisión colectiva, y también, alguna vez, a la traducción comparada, tan estimulante (pienso por ejemplo en nuestra versión de la «Oda a una urna griega» de

\footnotetext{
${ }^{1}$ Profesora de Filología Francesa, traductora y miembro del TTL.
} 
John Keats). Para mí, en todo momento, fue crucial la idea de Haroldo de Campos (uno de los grandes inspiradores y animadores, desde Brasil, de nuestro Taller) según la cual era preciso fundar lo que él llamaba «laboratorios de textos» para resolver el dilema entre las traducciones filológicamente correctas y las traducciones creativas ejemplares. Este ha sido siempre el gran debate. ¿No podrían conciliarse la dimensión creativa y el rigor filológico? Ya sabemos que la falta de rigor es la acusación central dirigida contra Pound, que se tomó unas «libertades» nunca aceptadas por los sinólogos o los provenzalistas, mientras que los creadores, por su parte, reprochan a los filólogos el ignorar la dimensión literaria de los textos y el operar sobre estos como cuerpos sin vida, como «letra muerta». Pues bien, siguiendo la idea de Haroldo de Campos, y también el estímulo de otros traductores excepcionales (por ejemplo, Juan Ramón Masoliver, a quien tuve la suerte de tratar en Barcelona), nuestro propósito fue desde un principio armonizar esos dos aspectos. La traducción rigurosa y creativa era posible.

Por supuesto, presidiéndolo todo estaba el principio de la «intraducibilidad» de la poesía, y cuando digo poesía no me refiero sólo, claro está, a los versos, sino a cualquier texto estéticamente complejo. Pero enfrentada a ese principio estaba, del mismo modo, la posibilidad de la re-creación, la reescritura, la reinvención de los textos, como demuestran numerosos casos en la modernidad literaria. Esa posibilidad ha sido siempre nuestra guía, nuestro objetivo estético. Por supuesto, no me corresponde juzgar los resultados (que, por lo demás, han de ser necesariamente variables, puesto que también los textos que hemos traducido en todos estos años presentan muy diversos grados de dificultad o de complejidad: no es lo mismo el grado de exigencia que requiere el Diccionario de lugares comunes, de Flaubert -uno de nuestros primeros trabajos-, o el Prefacio a Shakespeare, del doctor Johnson — también entre los primeros—, que los Tres poemas secretos, de Seferis, llenos de claves internas, «secretas», con una trama textual y sonora de una extraordinaria densidad, o los intrincados Cuadernos de Valéry). La dificultad no nos ha asustado nunca. Todo lo contrario. La «intraducibilidad» ha sido para nosotros una especie de acicate. De ahí que me guste recordar y citar a menudo la reflexión de Léon Robel según la cual «todo traductor, y sobre todo el traductor de poesía, debe ser un especialista de lo intraducible».

En cuanto al otro aspecto de tu pregunta, es decir, si las expectativas de 1995 se han cumplido o no, creo que la respuesta está contenida en lo que ya he comentado: se han logrado ampliamente, tanto por todo lo que hemos podido hacer hasta aquí como por el alcance de nuestras publicaciones. Lo digo sin triunfalismo alguno, ateniéndome sólo a una cierta ética del trabajo bien hecho. No entro, insisto, en ningún juicio crítico acerca de lo realizado; me refiero únicamente a la coherencia de los propósitos, a la claridad de los objetivos, y también — y quizá ante todo - al enorme placer que los textos nos han proporcionado, a la «alquimia del verbo» que se ha operado en cada texto que traducíamos.

CC: Quien haya seguido la trayectoria del TTL conoce bien no sólo el elevado volumen de publicaciones que de él han surgido, sino también su diversidad y calidad, como así lo atestiguan las 
numerosas y elogiosas reseñas que han recibido. Pero supongo que algunos proyectos se habrán quedado por el camino. ¿Qué podrías señalar sobre ello?

ASR: Este es otro aspecto de lo que vengo comentando. El hecho de que hayamos trabajado en diversos géneros (poesía, narrativa, ensayo, diario, aforismo) y en muy distintas lenguas (desde el portugués o el checo hasta el sueco o el polaco) no significa que todos nuestros proyectos hayan podido realizarse en el nivel deseado. Mejor dicho: realizarse hasta ahora, porque de hecho siguen en cartera distintos planes y se mantienen vivas algunas aspiraciones. El caso es que, tanto por su estructura de trabajo en grupo como por cuestiones puramente organizativas (un único encuentro semanal de unas tres horas a lo largo del curso académico), el Taller no ha podido llevar a cabo algunos proyectos frecuentemente comentados en nuestras reuniones. Me refiero por ejemplo a traducciones del árabe, que por una razón u otra hemos debido ir aplazando ante el compromiso de otras traducciones muy ambiciosas, y estoy pensando sobre todo en libros cuya traducción nos ha llevado varios años de trabajo, como los citados Cuadernos de Valéry o la amplia antología de los poetas metafísicos ingleses (todavía en curso de publicación), para no hablar de la versión de $E l$ puente, de Hart Crane, libro en el que tuvimos que invertir mucho más tiempo del inicialmente previsto, o la extensa antología de Mario Luzi... Todos ellos han sido trabajos de muy larga duración, realizados sin prisa, con su propio calendario, su propio tempo, a veces muy lento, valorando cada palabra, cada verso, cada alusión, cada matiz, e incluso deteniéndonos en el uso de tal o cual signo de puntuación. Eres testigo de cómo un solo verso, una sola frase, ha podido a veces ocuparnos durante horas, y no ya sólo en busca de la traducción más apropiada, sino también tratando de comprender bien el texto, de aprehenderlo en todos sus valores. Por eso he comentado alguna vez que el nuestro es un taller «rabínico», en el que la lectura y la interpretación de cada palabra resultan trascendentales. Naturalmente, todo esto ha requerido muchísimas horas de dedicación, y del mismo modo que, por razones de tiempo, no hemos podido hacer nada de lengua árabe hasta hoy, tampoco hemos tenido oportunidad de trabajar hasta la fecha en clásicos griegos y latinos, algo que formaba parte de nuestros planes iniciales (Propercio, Catulo). Esta es la razón por la que, además, algunos de nosotros hemos tenido que participar en diferentes proyectos de traducción al margen del Taller, porque éste, ante las limitaciones de tiempo, no podía asumirlos o integrarlos. En mi caso, por ejemplo, he trabajado en versiones de poesía japonesa en colaboración con Masafumi Yamamoto, y siempre he lamentado mucho que los resultados no pasaran por la revisión — para mí siempre tan efectiva — del trabajo en equipo del Taller. Lo mismo me ha ocurrido con las versiones de poesía eslovena, que he realizado con otra colaboradora del Taller, Laura Repovš, pero que, por idénticos motivos, no han podido ser objeto de la revisión del Taller. ¿Cómo interrumpir, por ejemplo, la serie de los poetas metafísicos ingleses — una labor que nos llevó cinco años - para ocuparnos de Masaoka Shiki o de una antología de poesía eslovena, proyectos que sin duda nos hubieran exigido, en cada caso, otros tantos años? Por suerte, hemos tenido el Boletín del TTL para ir dando a conocer algunas de esas traducciones llevadas a cabo al margen del Taller. Ha sido una manera de mostrar que lo realizado en él no es sino una parte de una actividad que, en cierto 
sentido, lo desborda. Y no hay que olvidar que, al mismo tiempo, hemos publicado tres extensos volúmenes de poesía moderna (De Keats a Bonnefoy, 2006; Ars poetica, 2011, y Las llamas sobre el agua, 2016), de manera paralela a los trabajos más extensos... Pero, como te decía, aún tenemos planes ambiciosos, como es una selección de pasajes de la Comedia de Dante, propuesta por José Juan Batista, y en la que empezaremos a trabajar una vez concluidas las tareas todavía en curso: el Diario 1931-1934 de Seferis, el libro Si la piedra florece del italiano Antonio Prete (poeta, traductor y crítico muy cercano a nuestro Taller) y Los labios y la sed del belga Yves Namur. En resumen: lo que se ha quedado por el camino no puede darse por definitivamente abandonado, y en todo caso nuestra estructura misma de trabajo y nuestro método han marcado de manera inevitable nuestras limitaciones.

CC: En otoño del 2011 comenzó a publicarse el Boletín del TTL con la voluntad de «dar una noticia más amplia y detallada de las actividades que realiza» y, al mismo tiempo, presentar algunos escritos teóricos que sustentan su práctica. En estos días ha visto la luz el número 26 y me consta que esta breve publicación trimestral llega a universidades, escritores y traductores de todo el mundo. ¿Te has planteado ampliar su extensión o disponer de un medio más actual —pienso en una página web, por ejemplo - que dé más visibilidad y accesibilidad a las distintas labores del Taller?

ASR: Sí, desde luego. La página web sería algo ideal para buscar y encontrar una mayor difusión. Confío en que no tardemos en crear esa página, en la que incluiríamos tanto la colección completa del Boletín del TTL como pequeñas muestras de nuestros trabajos en curso, discusiones críticas, artículos... El Taller, como bien sabes, genera multitud de notas, datos, comentarios que se realizan en el proceso mismo de traducción, algunos de los cuales valdría la pena reproducir como ejemplos de la «alquimia del verbo» de la que hablábamos antes, y que está en la base de toda traducción que se quiera al mismo tiempo rigurosa y creativa. El Boletín surgió en 2011, después de un largo período de reflexión acerca de cuál podría ser el medio más adecuado para difundir mejor nuestras tareas. Me decidí por la forma en que finalmente se edita porque se adapta de manera idónea al dinamismo que queríamos imprimir a la publicación, en la que caben reseñas y notas pero también artículos extensos, que aparecen en entregas sucesivas. Aunque todo gira en torno a la traducción, tienen cabida igualmente los textos teórico-críticos sobre literatura general, como por ejemplo, en el número 4, el bello ensayo de Judith Balso «Afirmación de la poesía. Cuestiones de método». Publicamos, además, adelantos de nuestros trabajos, como en el caso de la antología de los poetas metafísicos, contrastando nuestras versiones con las de otros traductores: así lo hicimos, por ejemplo, con el poema de John Donne «Elegie: Going to Bed», del que ofrecimos, junto a la versión española de Octavio Paz, la portuguesa de Augusto de Campos, y finalmente la nuestra, con el fin de que el lector pudiese establecer los nexos y las diferencias que considerase oportunos. Todo ello junto a una entrevista a Augusto de Campos y unos fragmentos del conocido libro de Antoine Berman sobre Donne (Pour une critique des traductions, 1995). 
Por cierto: esa página web a la que te refieres debería contener igualmente - como homenaje a su autor - el ensayo de Alejandro Cioranescu «El arte de la traducción», pieza maestra de un investigador y crítico excepcional que fue también un traductor excelente y que, desde la Universidad de La Laguna, realizó una amplia tarea en el ámbito de la literatura comparada, disciplina de la que ofreció en nuestra universidad el primer curso impartido en España. Siempre he visto los trabajos del TTL en una línea de continuidad con las enseñanzas que en materia de comparatismo desarrolló Cioranescu en La Laguna. Somos, por así decirlo, sus herederos, y de ahí que hayamos resaltado siempre con tanto empeño, en el Boletín, la estrecha vinculación entre comparatismo y traductología, asunto al que hemos dedicado varias notas y artículos. No hace mucho la italiana Marina Guglielmi subrayaba «el acto de la traducción que se oculta, en realidad, tras todo proceso de intercambio y comunicación», es decir, tras todo acto de comparación entre formas, géneros o modalidades literarias; en definitiva, tras toda transferencia o relación entre literaturas.

CC: En las distintas ocasiones en las que has aludido al nacimiento y al funcionamiento del TLL has insistido en que se trata de un grupo estable, pero también abierto en cuanto al grado de compromiso. ¿Cómo se alcanza ese sutil equilibrio entre la libertad de participación y la necesaria dinámica de grupo que garantice la coherencia y el rigor precisos en el manejo de las premisas y criterios que caracterizan la labor del Taller?

ASR: Reconozco que es un equilibrio difícil. El margen de libertad en cuanto a la participación en los trabajos del Taller por parte de cada uno de sus miembros es tan grande que a veces linda con el más puro individualismo antiautoritario. Pero, como en la mejor tradición anarquista, se trata de una ética, de una responsabilidad peculiar, asumida íntimamente, sin ninguna obligación, salvo la que uno mismo quiera adjudicarse. Todos los participantes aportan algo, de un modo u otro todos suman; si no, no hay tarea colectiva, que es la base del Taller. El grupo que tomó forma en 1995 era muy amplio. Pronto vimos que no todos los que se apuntaron al proyecto estaban dispuestos a mantener el grado de compromiso que se exigía; un grado considerable, dada la ambición de nuestros planes. Eso ya redujo un poco el número de participantes, que iban desde estudiantes hasta profesores, pasando por algunos bibliotecarios. En los meses que siguieron, el número se redujo aún más: el proyecto demandaba un esfuerzo que no todos podían o estaban dispuestos a realizar. En casi un cuarto de siglo desde que iniciamos nuestras tareas, puedes imaginar que hemos pasado por distintas fases, y en buena parte tú misma has asistido a ellas. Hemos logrado formar un grupo estable, pero no sin ciertas vicisitudes.

El Taller es un seminario permanente, y han pasado por él muchas personas interesadas en la traducción literaria. Unas han trabajado con nosotros largo tiempo, otras sólo durante un período determinado, o sólo en la traducción de un libro o de un autor concreto. Quiero destacar aquí, por encima de todo, la contribución de cuatro personas muy queridas, que fallecieron estando vinculadas al Taller y muy especialmente comprometidas con sus actividades: me refiero a Alberto Giordano, 
comparatista único, filólogo integral del que todos aprendimos mucho, y con quien realizamos nuestro primer trabajo de traducción colectiva, los Sonetos profanos y sacros de Paolo Valesio; Guy Rochel, lingüista notabilísimo y de extraordinaria sensibilidad literaria, que estuvo con nosotros casi desde el principio y que tradujo la novela de Bernard Noël El síndrome de Gramsci; Isabel García Gálvez, helenista, admirable conocedora del griego moderno, que realizó la versión de base de los Tres poemas secretos, de Seferis, y Margarita Gómez Sierra, que apenas tuvo tiempo de traducir con nosotros algunos poemas de Jules Laforgue. Los cuatro nos han dejado recuerdos imborrables.

Lo que tú llamas la «coherencia» y el rigor necesarios en cuanto a criterios y métodos se han mantenido siempre, a mi juicio, una vez que se han entendido esos criterios y se ha aceptado el método de la revisión colectiva, en el cual el respeto a las opiniones de los demás y la renuncia a cualquier orgullo o vanidad personal resultan esenciales, algo que tú misma has mencionado y valorado en alguno de tus artículos. Ya sabes que, en nuestro método de la traducción revisada, se impone siempre la opinión del grupo, pero el traductor o traductora que realiza el trabajo de base y firma el resultado final tiene libertad para tomar la última decisión en tal o cual aspecto o detalle que aún le suscite dudas; generalmente se respetan las opiniones del grupo, pero se concede al traductor firmante la oportunidad de decidir en tal o cual giro concreto más o menos problemático, como señal de respeto a su libertad y subjetividad de juicio. El método ha funcionado en todos los casos, y la treintena de libros publicados lo demuestra, además de los tres volúmenes de versiones de poesía moderna. Dicho esto, debo añadir que para mí, con el paso de los años, el Taller ha acabado siendo, por encima de todo, un grupo de amigos apasionados por la traducción literaria que se reúnen una vez a la semana durante unas horas para disfrutar de la mágica metamorfosis del paso de un texto de una lengua a otra, y que hacen de esa maravillosa transformación un placer compartido.

CC: Desde hace algunos años se ha fomentado la asistencia a las sesiones del Taller de alumnos de distintas especialidades filológicas, así como de estudiantes europeos que llegan a nuestra universidad a través del programa Erasmus. De este modo, se afianza la dimensión didáctica del Taller, de la que todos sus integrantes nos beneficiamos. ¿Cómo valoras la participación de todo este alumnado?

ASR: Haces bien en llamar la atención sobre este aspecto central del Taller. Ya sabes que, durante unos años, nuestros trabajos llegaron a tener el rango académico de asignatura optativa, y recibimos a un buen número de alumnos interesados. La experiencia fue ambivalente: había alumnos brillantes, sin duda, pero también alumnos mediocres que no hacían sino lastrar la tarea e impedirnos avanzar con el ritmo requerido; llegamos a aprender mucho de los primeros, muy especialmente de los más creativos. Asunto bien distinto es el de los estudiantes del programa Erasmus: en todos los casos, la experiencia ha sido especialmente interesante, porque si no siempre han sabido ser lo bastante creativos, por pura bisoñez, siempre han mostrado, en cambio, una gran dedicación y han entendido cabalmente el método de la traducción revisada, con el que se han identificado en buena medida, revelando una gran capacidad de colaboración y un interés fuera de lo común por el proceso 
de traducción, algo que en el caso de alumnos extranjeros tiene, por supuesto, una relevancia especial. No podremos olvidar, y es sólo un ejemplo, el caso de las alumnas polacas con las que tradujimos poemas de Czesław Miłosz, de Tadeusz Różewicz, de Zbigniew Herbert, de Adam Zagajewski... Su simpatía era contagiosa, y se desvivían por explicar determinado giro o tal o cual matiz semántico. Pero no todos nuestros colaboradores ocasionales o temporales han sido estudiantes: también hemos contado con poetas, traductores, profesores y escritores extranjeros que han querido sumarse a traducir a autores rumanos, checos, suecos, daneses, griegos, rusos... De todos esos colaboradores hemos aprendido mucho: nos han enseñado no sólo cuestiones de lengua, sino también dimensiones culturales nuevas y del todo desconocidas, una razón más - y no la menos importante, precisamente - para pensar que la traducción se halla en la base misma tanto de los procesos cognitivos como de cualquier fenómeno de comunicación. La traducción, en definitiva, no sólo como acto lingüístico, sino también como hecho portador de otras muchas «significaciones analógicas», como dice el viejo diccionario de Covarrubias...

CC: A partir de un determinado momento, como ya has dicho, el TTL se decantó por el método de la revisión colectiva de una versión más o menos literal realizada por uno o dos de sus miembros, que son los que luego firman la publicación. Aparte de su indiscutible efectividad, agilidad y rapidez frente a la traducción conjunta propiamente dicha que se practicó en los inicios del Taller, ¿da respuesta esta segunda modalidad al delicado problema de la autoría múltiple? ¿Las editoriales han planteado, en alguna ocasión, algún tipo de reparo?

ASR: Asunto importante, sí. Para mi sorpresa, nuestro primer método, el de la traducción colectiva o conjunta, presentaba dos serios problemas, el primero de orden práctico, y el segundo de tipo editorial. La traducción colectiva, tan apasionante, tan mágica en ciertos momentos $-\mathrm{y}$ que todavía practicamos en el Taller, pero sólo de manera muy ocasional—, puede llegar a ser, en cambio, de una lentitud desesperante, poco operativa en caso de textos extensos, algo en lo que yo había reparado ya en los talleres de Royaumont. La traducción revisada, una fórmula ideada en el TTL, fue un intento de solucionar ese problema, y sus resultados están a la vista. Pero la traducción colectiva, además, suscitaba en las editoriales ciertos recelos que no podíamos imaginar: pensaban, piensan, que se trata de una suerte de «experimento» creativo poco fiable, demasiado anárquico y, por tanto, lleno de «infidelidades», en el que no hay un criterio unificador... Impresión falsa e injusta, por supuesto, porque se ignora que la traducción colectiva exige ciertos acuerdos previos, ciertas pautas aceptadas por todos de antemano (el tipo de texto de llegada, su grado de «libertad», en el caso de la poesía si hay o no una propuesta métrica, etc.). Si te fijas bien, se trata de unos recelos parecidos a los que ciertas editoriales y ciertos traductores profesionales han mostrado a veces respecto a los «seminarios de traducción» de algunas universidades, una injusticia ya denunciada, entre otros, por André Lefevere... He abordado en otra ocasión este asunto, al examinar la relación entre la literatura comparada y la traducción literaria. No será preciso extenderse ahora sobre ello. De todo esto hablamos, como recordarás, en nuestra ponencia en el congreso de la 
Universidad de Bolonia, sede de Forlì, al que fuimos invitados en la primavera de 2012. Será preciso remitir aquí a lo que dijimos entonces y que se puede leer en el número 5 del Boletín del TTL (otoño de 2012).

CC: Por último, ¿quieres añadir algo en relación con los proyectos que en estos momentos tiene en perspectiva el TTL para los próximos años? A pesar de los inconvenientes de la traducción colectiva que acabamos de comentar, ¿se ha previsto volver en algún momento a esa práctica?

ASR: Ya hablamos antes, de paso, sobre los proyectos y trabajos en curso. Quisiera referirme de nuevo, sin embargo, a una de nuestras iniciativas más ambiciosas, la antología de poetas metafísicos ingleses en la que hemos estado trabajando durante cinco años, con poemas muy importantes que se traducen por vez primera al español, y que conforman la selección y traducción más extensa de estos creadores realizada hasta hoy en nuestra lengua. El libro está ya terminado, como sabes, y pendiente ahora tan sólo de la introducción crítica que me viene ocupando desde hace algún tiempo. Creo que por primera vez podrá verse en castellano, en toda su amplitud, la importancia decisiva de estos poetas esenciales para la historia de la poesía europea. Creo también que se trata de uno de esos trabajos en los que puede calibrarse idóneamente, además, el esfuerzo realizado por el TTL. Y en cuanto a la traducción colectiva, ya señalé que no la hemos abandonado del todo: hay algunas muestras, por ejemplo, en el tercer volumen de poesía moderna, Las llamas sobre el agua. Seguiremos recurriendo a ella en textos muy breves, tanto en poesía como en prosa: su magia es insustituible. Es como la creación de un poema colectivo, con toda su fuerza y su raro poder de encantamiento.

\section{Publicaciones acerca del TLL}

CAlabrese, G. (2012): «Il “Taller de traducción literaria”: un'alternativa per la didattica della traduzione poetica», en E. LANDONE, ed., Enlaces. Studi dedicati a Mariarosa Scaramuzza. Milán, LED Edizioni Universitarie, pp. 63-72 (versión española de A. SORRENTINO y J. DÍAZ ARMAS en Piedra y Cielo, II/2, 2013, pp. 29-36).

CurELl, C. (2013): «Reflexiones sobre la práctica colectiva de la traducción. Algunas versiones de poesía catalana y francesa moderna», Bulletin hispanique, 15-2, pp. 637-651.

MorAles, C. J. (2007): «Las posibilidades de la traducción en la poesía moderna», Nueva revista de política, cultura, arte, 109, pp. 112-117.

Rochel, G. (1998): «Poesía y traducción. Conversación con Andrés Sánchez Robayna», Cuadernos Hispanoamericanos, 576, pp. 63-73.

Rodríguez Refojo, A. (2005): «Diez años del Taller de Traducción Literaria de la Universidad de La Laguna», Piedra y Cielo, 3, pp. 22-28. 
Ruiz Casanova, J. F. (2014): «La traducción colectiva (o de autoría múltiple): de Toledo al Taller de La Laguna», en L. DoLFI, ed., La traduzione. Opere e autori del Novecento. Milán, MUP, pp. 17-37.

SÁnchez Robayna, A. (1996): «El Taller de Traducción Literaria», Diario 16, supl. «Culturas», 23 de noviembre.

- (2006): «Acerca del Taller de Traducción Literaria», Ínsula, 717, pp. 2-4.

- (2006): «Introducción», en A. SÁnchez RoBAynA, ed., De Keats a Bonnefoy. (Versiones de poesía moderna). Valencia, Pre-Textos, pp. 11-22.

- (2007): «Traducir, esa práctica», en J. DocE, ed., Poesía en traducción. Madrid, Círculo de Bellas Artes, pp. 201-239.

- (2008): «Traducir y ser traducido», en J. GÓMEZ MONTERO, ed., Nuevas pautas de traducción literaria. Madrid, Visor, pp. 183-197.

- (2011): «Introducción», en A. SÁNCHEZ RoBAYNA, ed., Ars poetica. (Versiones de poesía moderna). Valencia, Pre-Textos, pp. 9-20.

- (2013): «Más sobre la traducción colectiva», Boletín del Taller de Traducción Literaria de la Universidad de La Laguna, 7, pp. 1-2.

- (2016): «Introducción», en A. SÁNCHEZ ROBAYNA, ed., Las llamas sobre el agua. (Versiones de poesía moderna). Valencia, Pre-Textos, pp. 7-19.

SÁnchez Robayna, A. - DíAz ARMAs, J. - Curell, C. (2012): «La traducción colectiva: teoría y práctica», Boletín del Taller de Traducción Literaria de la Universidad de La Laguna, 5, pp. 14. 